\title{
Editorial
}

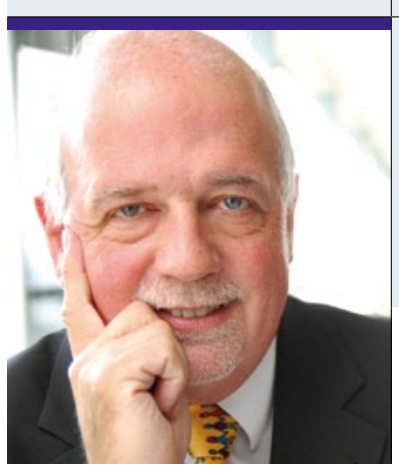

"Die ausschließliche Beschäftigung

mit Beatmungsproblemen tritt in den Hintergrund.

Neue Schwerpunkte tun sich auf."

\section{Frühgeborenenmedizin am Puls der Zeit}

"D ass Frühgeborene nicht atmen können, ist seit altersher bekannt", so lautete schon der Beginn meines Habilitationsvortrages vor 20 Jahren. Große, gerade auch Deutschland-basierte, Untersuchungen haben gezeigt, dass dies nur eingeschränkt gilt und führten zu einer minutiös ausgefeilten Beatmungsvermeidungsstrategie, auch bei extrem kleinen Frühgeboren. Atemhilfen im Sinne von CPAP („continuous positive airways pressure"), kombiniert mit einer nicht invasiven SurfactantTherapie, sind von Vorteil, auch bei den kleinsten Frühgeborenen. Sie unterstützen das Konzept einer sanften Frühgeborenenmedizin.

Die Frühgeborenenmedizin tritt damit in ganz neue Zonen. Die ausschließliche Beschäftigung mit Beatmungsproblemen tritt in den Hintergrund. Neue Schwerpunkte tun sich auf und müssen wissenschaftlich und klinisch beleuchtet werden (vgl. Beitrag S. xy). Die extrauterine Wachstumsretardierung, d. h. der Zustand, dass sehr unreife Frühgeborene der 24.-30. Schwangerschaftswoche heute am errechneten Termin immer noch nur um $2.500 \mathrm{~g}$ wiegen, muss angegangen werden, da die Langzeitfolgen für die neurologische Entwicklung und andere Morbiditätsrisiken ebenso groß wie bei intrauteriner Wachstumsretardierung sind. Mit neuen Ernährungsregimen gelingt das auch.

Der wesentliche Risikofaktor für extrem kleine Frühgeborene bleibt und wird zunehmend die Unreife des Abwehrsystems sein, insbesondere die intestinalen Barriere für Keime aller Art. Übliche Strategien der Krankenhaushygiene sind hier von nachrangiger Bedeutung, wie aktuelle Übersichtsarbeiten zeigen. Dieser Aspekt ist aber Medien und politischer Öffentlichkeit nur schwer vermittelbar. Die Skandale wegen angeblicher Hygieneprobleme in neonatologischen Abteilungen haben die bisher positive Erscheinung der Frühgeborenenmedizin in der Gesellschaft inzwischen ins Gegenteil verkehrt.

Selbst ethische Entscheidungen an der Grenze der Lebensfähigkeit werden mittlerweile öffentlich- keitswirksam (wie im Kölner Fall) infrage gestellt. Hier fühlen sich Neonatologen zunehmend alleine gelassen und das Fach als solches falsch repräsentiert - vor allem, weil in der Fläche die Partner in der Geburtshilfe fehlen.

Ausgewiesene Geburtshelfer, die sich hauptsächlich mit Frühgeborenenmedizin beschäftigen, werden zunehmend rar. Es ist ein dringender Wunsch und eine Hoffnung für die Zukunft, dass die so gut angelaufene Zusammenarbeit zwischen Geburtshelfern und Kinderärzten, wie sie in der Perinatalmedizin bisher für viele Bereiche der Medizin beispielgebend ist, als sinnvolles Konzept akzeptiert wird. Sie ist wichtig und wird immer wichtiger, nicht nur bei extrem prämaturen Kinder, sondern auch bei den späten Frühgeborenen, wo sehr fein über den optimalen Entbindungszeitpunkt gesprochen werden muss.

Einerseits sind Morbiditäts- und Mortalitätsrisiken vor der $39+0$. Gestationswoche für Kinder bei elektiver oder auch nur primärer Sectio höher, andererseits müssen sie immer im Verhältnis zum möglichen, intrauterinen Fruchttod diskutiert werden. Ein kollegiales, ja freundschaftliches interdisziplinäres Gespräch mit häufigen Kontakten zwischen Geburtshelfern und Neonatologen ist nicht weniger wertvoll.

Bei den niedrigen Geburtenzahlen und dem fehlenden Nachwuchs in der Geburtshilfe ist das ein weiteres, wichtiges Argument für eine Zentralisierung dieser Risikogeburten - natürlich immer vor dem primären Hintergrund, Frühgeburten zu vermeiden. Dabei wundert es durchaus, dass in Deutschland die Progesteron- und 17-OHP-Therapie zur Vermeidung von Frühgeburtlichkeit bei bestimmten Indikationsgruppen so zurückhaltend angenommen wird.

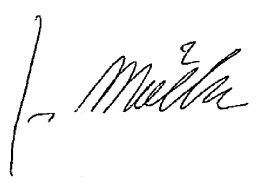

